\title{
Low Serum Mannose Binding Lectin (MBL) Levels and -221 YX Genotype of MBL2 Gene Are Susceptible to Neonatal Sepsis in the Chinese Han Population
}

\author{
Heng Xue, ${ }^{1,2}$ Xiaoguang Xue, ${ }^{1}$ Changyi Yang, ${ }^{3,}{ }^{*}$ Qingquan Chen, ${ }^{2}$ Ni Lin, ${ }^{2}$ Yujun Lin, ${ }^{4}$ Min Chen,,${ }^{, *}$ \\ Dongdong Rao, ${ }^{1}$ and Huizi Lin $^{3}$ \\ ${ }^{1}$ Department of Laboratory Medicine, Fujian Provincial Maternity and Children Hospital, Affiliated Hospital of Fujian Medical University, Fuzhou, China \\ ${ }^{2}$ Department of Laboratory Medicine, Medical Technology and Engineering College, Fujian Medical University, Fuzhou, China \\ ${ }^{3}$ Department of Neonatology, Fujian Provincial Maternity and Children Hospital, Affiliated Hospital of Fujian Medical University, Fuzhou, China \\ ${ }^{4}$ The fourth ward, Fuzhou Tuberculosis Prevention and Cure Hospital, Fuzhou, China \\ "Corresponding authors: Changyi Yang, 18 Daoshan Road, Fuzhou, 350001, Fujian, China. Tel: +86-059187555474, E-mail: cyyang996@qq.com; \\ Min Chen, 88 Jiaotong Road, Fuzhou, 350004, Fujian, China. Tel: +86-059183569250, E-mail: cmjy503@163.com
}

Received 2016 October 23; Revised 2016 December 11; Accepted 2017 January 13.

\begin{abstract}
Background: The diagnosis of neonatal sepsis remains a challenge as the condition lacks early clinical signs and reliable biomarkers.

Objectives: The aim of our study was to determine whether serum mannose binding lectin (MBL) levels and genotypes of MBL2 gene could be used as markers for predicting neonatal sepsis in the Chinese Han population.

Methods: This prospective study was hospital-based in design. 48 neonates with clinical signs and symptoms of septicemia (study group), and 96 infants with no infection (control) were included. All the neonates are Chinese Han descent. MBL2 promoter polymorphisms at positions $-550,-221$ and +4 were analyzed by direct sequencing, and serum MBL levels were estimated by enzyme-linked immunesorbent assay.

Results: Frequencies of genotype -221 YX were significantly higher in the study group (45.8\%) compared with the control group (15.60\%; $\mathrm{P}=0.00009$ ). The median serum MBL level was found to be significantly lower in infants who had the -221YX genotype (214.54 ng/mL) compared with those who had the -221YY genotype $(597.85 \mathrm{ng} / \mathrm{mL} ; \mathrm{P}<0.0001)$. Additionally, the median of serum MBL was significantly lower in infants with septicemia $(289.65 \mathrm{ng} / \mathrm{mL})$ than in controls $(597.75 \mathrm{ng} / \mathrm{mL} ; \mathrm{P}=0.041)$. According to ROC analysis, the cutoff value of MBL concentration $\leq 384.60(\mathrm{ng} / \mathrm{mL})$ had a sensitivity of 0.806 and a specificity of 0.777 for predicting sepsis.

Conclusions: This study suggested that serum MBL and the -221 YX genotype of the MBL2 gene might be predisposed factors for sepsis in the Chinese Han population.
\end{abstract}

Keywords: Genotype, MBL, Neonatal Sepsis, Polymorphism, Susceptibility

\section{Background}

Neonatal sepsis, a clinical syndrome of systemic infection, remains a common cause of morbidity and mortality in newborn infants, especially in developing countries (1). Timely diagnosis and treatment for septicemia is essential in neonates, since a delay in treatment may lead to a troublesome outcome (2). However, the diagnosis of neonatal sepsis is challenging because the clinical signs including changes in body temperature and tachycardia are nonspecific, and there is no single ideal reliable marker available for the diagnosis (3).

Common laboratory tests used for the diagnosis of sepsis are white blood count, absolute neutrophil count, Creactive protein (CRP) and procalcitonin estimation $(2,4$, $5)$. But these parameters have low sensitivity and are non- specific (3). Although bacteriologic results are the gold standard for the diagnosis of systemic infection, they have certain flaws such as low sensitivity, high incidence of falsenegative results and long test times (3). Besides, it is impractical to collect large volumes of blood sample for serial blood culture from infants. So, new laboratory methods including reliable markers are needed so that the diagnosis and treatment can be started without delay.

Mannose binding lectin (MBL) is one of the most pivotal factors in innate immunity which acts as the first line of defense against infection in newborns (6). Studies have found that low serum MBL, usually caused by gene variation, has been related to more frequent and severe infections (6-9). The associations between MBL2 gene polymorphisms, circulating MBL levels, and susceptibility and out- 
comes in sepsis have been reported in several studies but have shown conflicting results (10-13). Moreover, the concentrations of MBL may be diverse in patients with different ethnicities and ages (14). And little is known about the relationship between MBL and neonatal sepsis in the Chinese Han population.

\section{Objectives}

The aim of our study was to determine whether serum MBL levels and genotypes of MBL2 gene could serve as markers for predicting neonatal sepsis in the Han population, MBL levels and MBL2 promoter polymorphisms at positions -550, -221 and +4, known as variants $\mathrm{H} / \mathrm{L}$ (rs11003125), X/Y (rs7096206) and P/Q (rs7095891) were investigated.

\section{Methods}

This study subjects consisted of 144 newborns treated at Fujian Provincial Maternity and Children Hospital, between January 2013 and December 2015. The study protocol and informed consent were approved by the Fujian Medical University ethics committee.

The inclusion criteria were as follows: 1) infants younger than 28 days post-partum; 2) hospitalized in the neonatal ward; and 3) diagnosed with sepsis. Diagnostic criteria for sepsis (15) are signs and symptoms of inflammation plus infection with hyper- or hypothermia (rectal temperature $>38.5$ or $<35^{\circ} \mathrm{C}$ ), tachycardia (may be absent in hypothermic patients), and at least one of the following indications of altered organ function: altered mental status, hypoxemia, increased serum lactate level, or bounding pulses.

The exclusion criteria were newborns with congenital anomalies, chromosomal abnormalities, or inborn errors of metabolism, and those who were already receiving parenteral antibiotic at the time of sepsis evaluation, or had just undergone surgery, or had no parental consent.

Neonates who were free from any clinical evidence of sepsis (i.e., no sepsis evaluation undertaken, no antibiotics administered) and were matched with the study group regarding gestational age and sex were selected as controls. The controls were collected at the same period as the study group.

The clinical characteristics and laboratory data of newborns were recorded, including gestational age, sex, birth weight, and laboratory parameters such as complete blood cell, differential white blood cell, platelet counts, and CRP. Blood samples were obtained and kept at $-20^{\circ} \mathrm{C}$ until DNA was extracted. Sera were separated immediately and stored at $-80^{\circ} \mathrm{C}$ until MBL enzyme-linked immunosorbent assay (ELISA) was done.
Genomic DNA was extracted from blood specimens, as described (16). The fragment of MBL2 gene promoter was amplified by polymerase chain reaction using the upstream primer 5'-AGGAGGATTCAAGGCAAGT-3' and the downstream primer 5'-CCAGAGAATGAGAGCTGAA-3'. Based on previous literature, the polymorphisms (rs11003125, rs7096206 and rs7095891) in the promoter region of MBL gene were verified by direct sequencing. The experimental conditions were reported previously (17) and the detail amplification parameters were as follows: $95^{\circ} \mathrm{C}$ for 5 minutes, 30 cycles of $95^{\circ} \mathrm{C}$ for 30 seconds, $55^{\circ} \mathrm{C}$ for 30 seconds, and $72^{\circ} \mathrm{C}$ for 1 minute, with a final extension step at $72^{\circ} \mathrm{C}$ for 10 minutes. The PCR products were purified and sequenced as described (16).

The serum concentration of MBL was detected by ELISA (Human MBL ELISA Kit; Ameko, Shanghai, China) according to the manufacturer's protocol in the kit.

\subsection{Statistical Analysis}

All data were analyzed with SPSS version 18.0 (SPSS Inc., Chicago, IL, USA). Continuous variables are expressed as median (QL-QU). The Kruskal-Wallis $\mathrm{H}$ test was used for comparing serum MBL levels among different genotypes. The Mann-Whitney U test was used for comparison within neonates with sepsis and controls. Categorical variables between groups were analyzed using Chi-square test. The associations between serum MBL concentration and sepsis were estimated by unconditional logistic regression and adjusted for birth weight. Receiver operating characteristic (ROC) curves were used to find out the optimal cut-off value of MBL for predicting sepsis. Corresponding diagnostic characteristics (sensitivity, specificity, positive predictive value, negative predictive value and area under curve) were given. For all tests, a two-sided Pvalue $<0.05$ was considered significant.

\section{Results}

\subsection{Baseline Characteristics of the Study Neonates}

The baseline characteristics of the infants are summarized in Table 1 . A statistically significant difference was observed between the median of birth weight in the study group (2570 g) in comparison with the control group (3100 $\mathrm{g}, \mathrm{P}=0.006)$. There were no significant deviations in the distribution of other clinical characteristics between the neonates with sepsis and controls ( $\mathrm{P}>0.05)$. Some regular laboratory parameters are shown in Table 2 . Our results showed that total leukocytes count, absolute neutrophil count, and CRP were significantly higher in infants with sepsis than in control group $(\mathrm{P}=0.009,0.025$ and 0.005 , respectively). However, platelet counts were slightly lower in neonates with sepsis than in control group. 
Table 1. Clinical Characteristics of Neonates with Sepsis and Controls

\begin{tabular}{|c|c|c|c|}
\hline Characteristics & $\begin{array}{l}\text { Neonates with } \\
\text { Sepsis }(n=48)\end{array}$ & Controls $(n=96)$ & P Value \\
\hline Birth weight, $g$ & $2570(1727-3346)$ & $3100(2249-3575)$ & 0.006 \\
\hline Gestational age, w & $38.2(33.7-40.3)$ & $37.3(36.1-39.3)$ & 0.811 \\
\hline Male, \% & $28(58.3)$ & $46(47.9)$ & 0.238 \\
\hline \multicolumn{4}{|l|}{ Mode of delivery } \\
\hline Vaginal, \% & $28(58.3)$ & $57(59.4)$ & 0.905 \\
\hline \multicolumn{4}{|l|}{$\begin{array}{l}\text { Maternal risk } \\
\text { factors }\end{array}$} \\
\hline $\begin{array}{l}\text { Prolonged } \\
\text { rupture of } \\
\text { membranes } \\
(\geq 18 \mathrm{~h}), \%\end{array}$ & $8(16.7)$ & $12(12.5)$ & 0.496 \\
\hline $\begin{array}{l}\text { Multiple } \\
\text { births, \% }\end{array}$ & $7(14.6)$ & $10(10.4)$ & 0.465 \\
\hline $\begin{array}{l}\text { Antenatal } \\
\text { steroid use, } \\
\%\end{array}$ & $6(12.5)$ & $9(9.4)$ & 0.563 \\
\hline Apgar score & $10(9-10)$ & $10(9-10)$ & 0.107 \\
\hline
\end{tabular}

Table 2. Laboratory Parameters of Neonates

\begin{tabular}{lccc}
\hline $\begin{array}{l}\text { Laboratory } \\
\text { Parameters }\end{array}$ & $\begin{array}{l}\text { Neonates with } \\
\text { Sepsis }(\mathbf{n}=\mathbf{4 8})\end{array}$ & Controls $(\mathbf{n}=\mathbf{9 6})$ & P Value \\
\hline TLC, 10/L & $12.71(8.83-17.45)$ & $10.27(8.88-12.32)$ & 0.009 \\
\hline $\begin{array}{l}\text { ANC, 10/L } \\
\text { Platelet count, } \mathbf{1 0}\end{array}$ & $206(146-255)$ & $233(203-284)$ & 0.049 \\
$\mathbf{L}$ & $0.85(0.50-6.59)$ & $0.50(0.50-0.53)$ & 0.005 \\
\hline CRP, $\mathbf{~ m g / L ~}$ & & & 0.025 \\
\hline $\begin{array}{l}\text { Abbreviations: ANC, absolute neutrophil count; CRP, C-reactive protein; TLC, to- } \\
\text { tal leukocytes count. }\end{array}$ & & \\
\hline
\end{tabular}

4.2. -221 YX Genotype of MBL2 Gene was Predisposed to Neonatal Sepsis

MBL2 promoter polymorphisms known as $\mathrm{H} / \mathrm{L}$ (rs11003125), X/Y (rs7096206) and P/Q (rs7095891) were confirmed by direct sequencing (Figure 1). The genotype distribution of rs11003125 in the infants was not calculated, because its genotype frequency was not in agreement with the Hardy-Weinberg equilibrium in neonates with sepsis. The genotype frequencies of both rs7096206 and rs7095891 polymorphisms were in agreement with the Hardy-Weinberg equilibrium in all groups analyzed. The alleles and genotypes distributions of rs7096206 and rs7095891 in both neonates with sepsis and controls are summarized in Table 3. Frequencies of genotype -221 YX were significantly higher in the study group (45.8\%) compared with the control group (15.60\%; P $=0.00009)$. And the sensitivity, specificity, positive predictive value and negative predictive value for predicting the sepsis were $0.458,0.840,0.595$ and 0.757 , respectively. These data indicate that the -221 YX genotype of MBL2 is susceptible to neonatal sepsis. And frequencies of genotype -221 YY were significantly lower in the study group (52.1\%) compared with the control group (72.9\%; $\mathrm{P}=0.013)$, which reveals that the -221 YY genotype of MBL2 might be a protective factor for neonatal sepsis. However, no significant differences were observed in allelic and other genotype frequency distributions for rs7096206 and rs7095891 between the neonates with sepsis and controls.

\subsection{Low Serum MBL was Positively Related to Neonatal Sepsis}

In order to know whether genotypes of rs7096206 and rs7095891 can affect serum MBL levels in neonates among the Chinese Han population, the serum MBL concentrations were measured. Our results showed that the median serum MBL concentrations of the -221YX genotype (214.54 $\mathrm{ng} / \mathrm{mL}$ ) and the -221XX genotype (363.87 $\mathrm{ng} / \mathrm{mL}$ ) were significantly lower when comparing to the -221YY genotype (597.85 ng/mL) (Figure 2A). But there was no obvious association between the median serum MBL concentrations of -221YX genotype and -221XX genotype. Moreover, we did not observe any significant differences for median MBL levels of $+4 \mathrm{PP}$ genotype when comparing with $+4 \mathrm{PQ}$ genotype. We did not compare the MBL levels of $+4 \mathrm{QQ}$ genotype with other genotypes, because there was only one infant with $+4 \mathrm{QQ}$ genotype (Figure $2 \mathrm{~B}$ ). Because the median birth weight of the study group was lower than control, which may affect several protein expressions, including MBL. So, in order to remove the effect of birth weight on MBL levels, the unconditional logistic regression was used to analyze he association between serum MBL concentration and sepsis with adjustment for birth weight. The median serum MBL concentration was significantly lower in neonates with sepsis $(289.65 \mathrm{ng} / \mathrm{mL})$ than in controls (597.75 ng/mL; $\mathrm{P}=0.041$ ) with adjustment for birth weight (Figure 2C). And the cutoff value of serum MBL concentration $\leq 384.60(\mathrm{ng} / \mathrm{mL})$ for predicting sepsis had a sensitivity of 0.806 and a specificity of 0.777 . The area under the curve was 0.844 (95\% confidence interval 0.775 - 0.913). Corresponding positive predictive value and negative predictive value were 0.604 and 0.904 , respectively (Figure 3).

\section{Discussion}

In this study, we investigated the association among genotypes of mannose binding lectin-2 (MBL2) gene, serum MBL levels and neonatal sepsis in the Chinese Han population. Our results showed that frequencies of genotype -221 YX were significantly higher in neonates with sepsis compared to controls. The median serum MBL concentrations 
Figure 1. Representative DNA Sequencing Analysis for MBL2 Promoter Polymorphisms

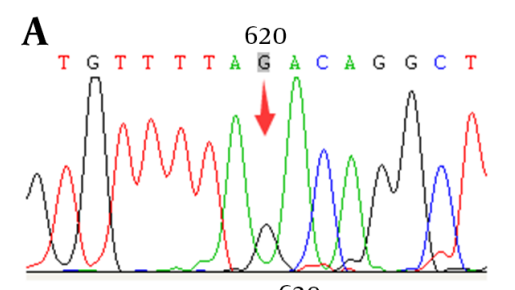

B $T$ T
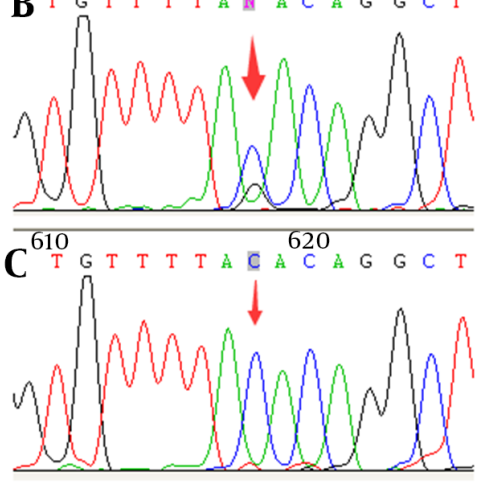

rs11003125
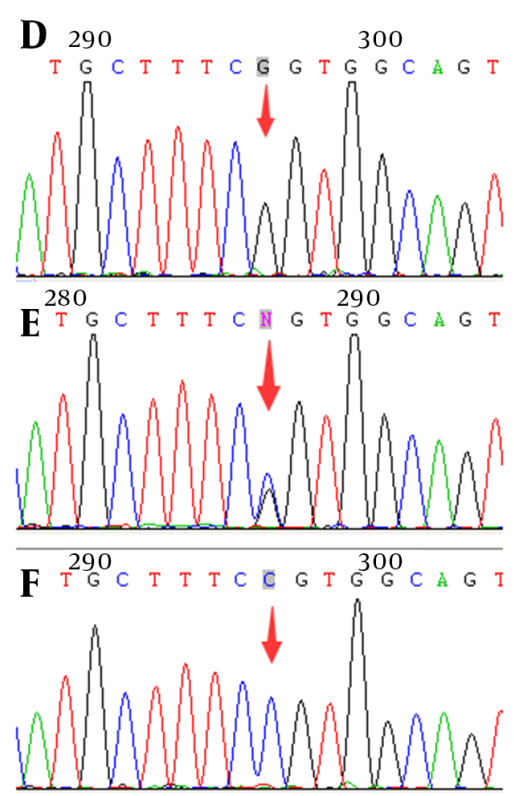

rs7096206
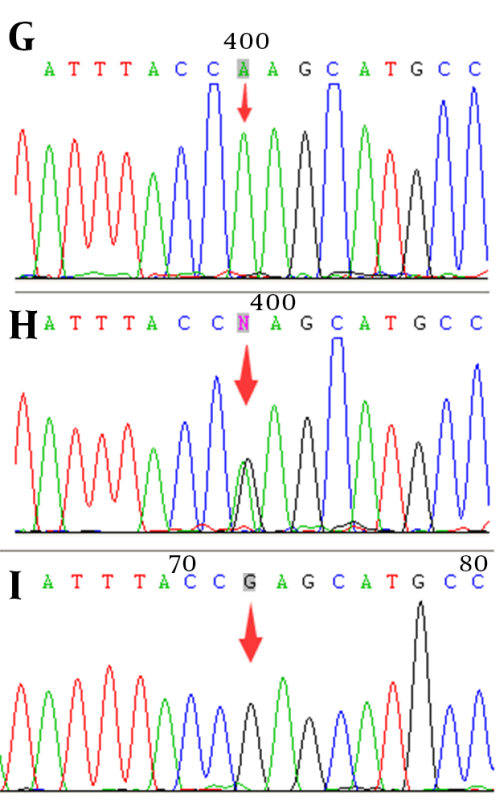

rs7095891

A-C, genotypes of rs11003125; D - F, genotypes of rs7096206; G - I, genotypes of rs7095891.

Table 3. The Alleles and Genotypes Distributions of rs7096206 and rs7095891 in Both Neonates with Sepsis and Controls

\begin{tabular}{|c|c|c|c|c|c|}
\hline Polymorphisms & Neonates with Sepsis & Controls & OR & 95\%CI & P Value \\
\hline rs7096206 & & & 1.40 & $0.78-2.51$ & \\
\hline $\mathrm{Y}$ & $72(75.0)$ & $155(80.7)$ & & & 0.262 \\
\hline $\mathrm{X}$ & $24(25.0)$ & $37(19.3)$ & & & \\
\hline $\mathrm{Y} / \mathrm{Y}$ & $25(52.1)$ & $70(72.9)$ & & & 0.0002 \\
\hline $\mathrm{Y} / \mathrm{X}$ & $22(45.8)$ & $15(15.6)$ & & & \\
\hline $\mathrm{X} / \mathrm{X}$ & $1(2.1)$ & $11(11.5)$ & & & \\
\hline rs7095891 & & & 0.47 & $0.20-1.08$ & \\
\hline $\mathrm{P}$ & $84(87.5)$ & $180(93.8)$ & & & 0.07 \\
\hline Q & $12(12.5)$ & $12(6.2)$ & & & \\
\hline $\mathrm{P} / \mathrm{P}$ & $37(77.1)$ & $84(87.5)$ & & & 0.145 \\
\hline $\mathrm{P} / \mathrm{Q}$ & $10(20.8)$ & $12(12.5)$ & & & \\
\hline $\mathrm{Q} / \mathrm{Q}$ & $1(2.1)$ & $0(0.0)$ & & & \\
\hline
\end{tabular}

${ }^{\mathrm{a}}$ Values are expressed as No. (\%).

of -221YX genotype was lower than -221YY genotype. Moreover, the median serum MBL concentration was also significantly lower in neonates with sepsis than in controls. Our results indicate that low serum MBL levels and-221 YX genotype of MBL2 gene might be novel markers for predicting neonatal sepsis in the Chinese Han population.

MBL is a critical factor in the innate immune system and provides the first line of protection against infection in neonates (6). Although many polymorphisms of MBL2 gene were investigated, only limited polymorphisms have 


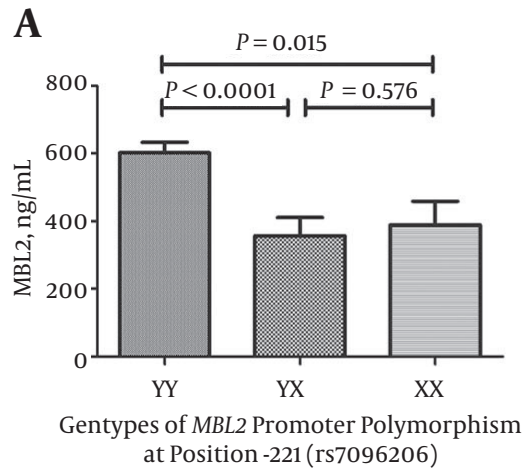

B

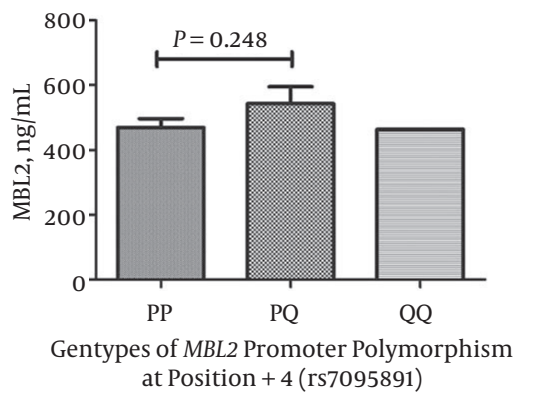

C

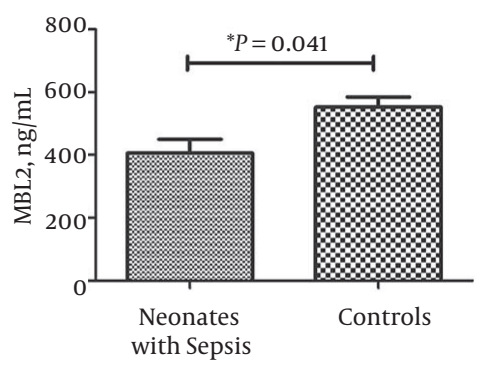

Sera were tested for MBL concentration by ELISA. A, MBL concentrations in different genotypes of rs7096206; B, MBL concentrations in different genotypes of rs7095891; C, MBL concentrations in neonates with sepsis and controls. *Data were calculated by unconditional logistic regression and adjusted for birth weight.

Figure 3. Receiver Operating Characteristic Curve for MBL Concentration Between Neonates with Sepsis and Controls

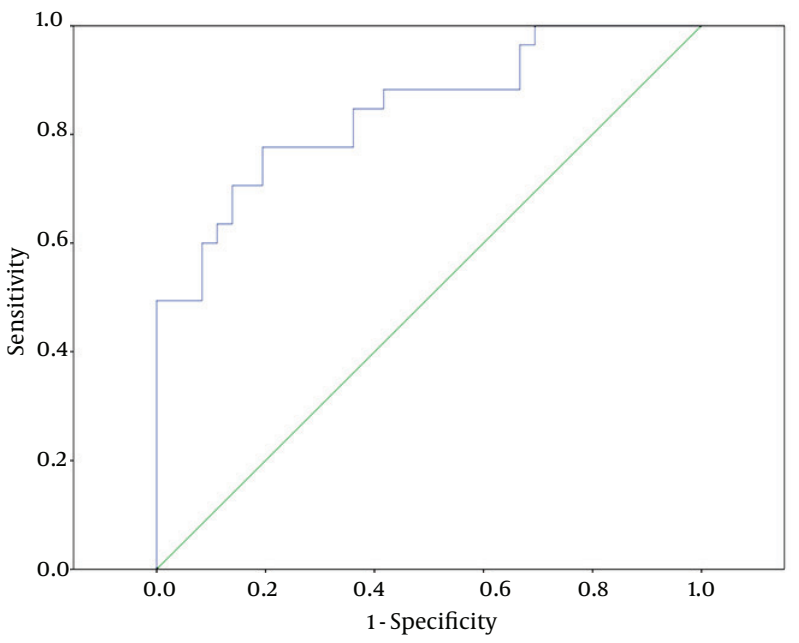

$\overline{\text { Acutoff value for serum MBL concentration } 384.60(\mathrm{ng} / \mathrm{mL}) \text { had a sensitivity of } 0.806}$ and a specificity of 0.777 . The area under the curve was 0.844 (95confidence interval 0.775 - 0.913).

functional relevance. And variations in the promoter region may affect binding of transcription factors leading to a decrease in the expression of MBL, which then impairs the function of the innate immune system and increases susceptibility to several infections (18). In the present study, we found that frequencies of genotype -221 YX were significantly higher in neonates with sepsis compared to controls and the median serum MBL concentration of the -221YX genotype was much lower than the -221YY genotype in the Chinese Han population. Our results indicate that the-221YX genotype of MBL2 may be used as a biomarker for predicting sepsis. Supporting our observation are the findings of Das and Panda (19), who reported that the -221YX genotype had low plasma MBL levels and was associated with increased susceptibility to infection. A similar conclusion was made by Prencipe et al. (20) who found that the 221YY genotype was associated with high MBL serum levels.

MBL can bind and opsonize various pathogens such as bacteria, viruses and parasites, which play a pivotal role in protecting against infection. In agreement with this theory, our study found that low serum MBL level was positively correlated with neonatal sepsis. Our data were in line with the study of Auriti et al. (21) and Liu and Ning (22), who reported that low MBL levels increased the risk of nosocomial sepsis. Our results were also concordant with the study of Gao et al. (14), who described that sepsis in the infant subgroup had considerably lower MBL level than those in the controls in Asians and Caucasians. But our results were in apparent contrast to the high MBL expression reported in the neonates with necrotizing enterocolitis (20). The discrepancies between these data and our results may be related to the pattern of MBL production. MBL is usually expressed by hepatocytes, but researchers also found that it has a strong expression in enterocytes, endothelial cells and histiocytes of the small intestine and colon (20). Therefore, when there are severe lesions in intestine such as necrotizing enterocolitis, MBL may be expressed at higher levels and result in higher rate of detection in serum.

In conclusion, this is the first study to our knowledge that investigated the association among MBL2 genotype, serum MBL concentration, and neonatal sepsis in the Chinese Han population. We found that low serum MBL level and -221 YX genotype of MBL2 gene was positively related to neonatal sepsis. Low serum MBL level and -221 YX genotype 
of MBL2 gene might be predisposing factors for neonatal sepsis in the Chinese Han population. Although the number of cases in this article is still limited, our findings add to the knowledge that the -221 YX genotype of MBL2 can decrease the expression of MBL, which may be involved in the pathway causing neonatal sepsis in the Chinese Han population.

\section{Acknowledgments}

This study was financially supported by grants from natural science foundation of Fujian province (Nos. 2014J01337, 2014J01338 and 2013J01317) and the Professor Foundation of Fujian Medical University (No. JS08008). We thank Ms. Jennifer Kilbourn for language editing.

\section{References}

1. Lawn JE, Cousens S, Zupan J, Lancet Neonatal Survival Steering T. 4 million neonatal deaths: when? Where? Why? Lancet. 2005;365(9462):891-900. doi: 10.1016/S0140-6736(05)71048-5. [PubMed: 15752534].

2. Adib M, Bakhshiani Z, Navaei F, Saheb Fosoul F, Fouladi S, Kazemzadeh H. Procalcitonin: a reliable marker for the diagnosis of neonatal sepsis. Iran J Basic Med Sci. 2012;15(2):777-82. [PubMed: 23493845].

3. Krishnaveni P, Vanitha M, Pradeep G. Estimation of serum amyloid A protein in neonatal sepsis: a prospective study. Int J Med Sci Public Health. 2016;5(8):1665. doi:10.5455/ijmsph.2016.06122015282.

4. Angeletti S, Battistoni F, Fioravanti M, Bernardini S, Dicuonzo G. Procalcitonin and mid-regional pro-adrenomedullin test combination in sepsis diagnosis. Clin Chem Lab Med. 2013;51(5):1059-67. doi: 10.1515/cclm-2012-0595. [PubMed: 23072859].

5. Zurek J, Vavrina M. Procalcitonin Biomarker Kinetics to Predict Multiorgan Dysfunction Syndrome in Children With Sepsis and Systemic Inflammatory Response Syndrome. Iran J Pediatr. 2015;25(1):e324. doi: 10.5812/ijp.324. [PubMed: 26199699].

6. de Benedetti F, Auriti C, D’Urbano LE, Ronchetti MP, Rava L, Tozzi A, et al. Low serum levels of mannose binding lectin are a risk factor for neonatal sepsis. Pediatr Res. 2007;61(3):325-8. doi: 10.1203/pdr.ob013e318030d12f. [PubMed: 17314691].

7. Ozkan H, Koksal N, Cetinkaya M, Kilic S, Celebi S, Oral B, et al. Serum mannose-binding lectin (MBL) gene polymorphism and low MBL levels are associated with neonatal sepsis and pneumonia. J Perinatol. 2012;32(3):210-7. doi:10.1038/jp.2011.79. [PubMed: 21681178].

8. Wahab Mohamed WA, Saeed MA. Mannose-binding lectin serum levels in neonatal sepsis and septic shock. J Matern Fetal Neonatal Med. 2012;25(4):411-4. doi: 10.3109/14767058.2011.582903. [PubMed: 21631240].

9. Giubergia V, Salim M, Fraga J, Castiglioni N, Sen L, Castanos C, et al. Post-infectious bronchiolitis obliterans and mannose-binding lectin insufficiency in Argentinean children. Respirology. 2015;20(6):982-6. doi: 10.1111/resp.12547. [PubMed: 25939617].

10. Mills TC, Chapman S, Hutton P, Gordon AC, Bion J, Chiche JD, et al. Variants in the Mannose-binding Lectin Gene MBL2 do not Associate With Sepsis Susceptibility or Survival in a Large European Cohort. Clin Infect Dis. 2015;61(5):695-703. doi: 10.1093/cid/civ378. [PubMed: 25969530].

11. Zhang AQ, Yue CL, Pan W, Gao JW, Zeng L, Gu W, et al. Mannosebinding lectin polymorphisms and the risk of sepsis: evidence from a meta-analysis. Epidemiol Infect. 2014;142(10):2195-206. doi: 10.1017/S0950268813003361. [PubMed: 24398289].

12. Frakking FN, Brouwer N, van Eijkelenburg NK, Merkus MP, Kuijpers TW, Offringa $\mathrm{M}$, et al. Low mannose-binding lectin (MBL) levels in neonates with pneumonia and sepsis. Clin Exp Immunol. 2007;150(2):255-62. doi: 10.1111/j.1365-2249.2007.03479.x. [PubMed: 17711490].

13. Ozdemir O, Dinleyici EC, Tekin N, Colak O, Aksit MA. Low-mannosebinding lectin levels in susceptibility to neonatal sepsis in preterm neonates with fetal inflammatory response syndrome. J Matern Fetal Neonatal Med. 2010;23(9):1009-13. doi:10.3109/14767050903551418. [PubMed: 20067428].

14. Gao DN, Zhang Y, Ren YB, Kang J, Jiang L, Feng Z, et al. Relationship of serum mannose-binding lectin levels with the development of sepsis: a meta-analysis. Inflammation. 2015;38(1):338-47. doi: 10.1007/s10753014-0037-5. [PubMed: 25323207].

15. Levy MM, Artigas A, Phillips GS, Rhodes A, Beale R, Osborn T, et al Outcomes of the Surviving Sepsis Campaign in intensive care units in the USA and Europe: a prospective cohort study. Lancet Infect Dis. 2012;12(12):919-24. doi: 10.1016/S1473-3099(12)70239-6. [PubMed 23103175].

16. Chen QQ, Dong F, Chen M, Gao F, Liu QC. Arg194-Arg399 haplotype of XRCC1 gene is susceptible to lung cancer in the Han population. EurJ Inflam. 2016;14(1):27-33.

17. Chen Q, Xue H, Chen M, Gao F, Xu J, Liu Q, et al. High serum trypsin levels and the-409T/T genotype of PRSS1 gene are susceptible to neonatal sepsis. Inflammation. 2014;37(5):1751-6. doi:10.1007/s10753-014-9904-3. [PubMed: 24777884].

18. Zinyama-Gutsire RB, Chasela C, Madsen HO, Rusakaniko S, Kallestrup $\mathrm{P}$, Christiansen $\mathrm{M}$, et al. Role of mannose-binding lectin deficiency in HIV-1 and schistosoma infections in a rural adult population in Zimbabwe. PLoS One. 2015;10(4):e0122659. doi: 10.1371/journal.pone.0122659. [PubMed: 25830474].

19. Das BK, Panda AK. MBL-2 polymorphisms (codon 54 and Y-221X) and low MBL levels are associated with susceptibility to multi organ dysfunction in P. falciparum malaria in Odisha, India. Front Microbiol. 2015;6:778. doi: 10.3389/fmicb.2015.00778. [PubMed: 26284055].

20. Prencipe G, Azzari C, Moriondo M, Devito R, Inglese R, Pezzullo M, et al. Association between mannose-binding lectin gene polymorphisms and necrotizing enterocolitis in preterm infants.JPediatr Gastroenterol Nutr. 2012;55(2):160-5. doi: 10.1097/MPG.0b013e31824e5f7a. [PubMed: 22331020].

21. Auriti C, Prencipe G, Inglese R, Azzari C, Ronchetti MP, Tozzi $A$, et al. Role of mannose-binding lectin in nosocomial sepsis in critically ill neonates. Hum Immunol. 2010;71(11):1084-8. doi: 10.1016/j.humimm.2010.08.012. [PubMed: 20732365].

22. Liu L, Ning B. The role of MBL2 gene polymorphism in sepsis incidence. Int J Clin Exp Pathol. 2015;8(11):15123-7. [PubMed: 26823854]. 